JOURNAL OF THEORETICAL

AND APPLIED MECHANICS

59, 2, pp. 307-317, Warsaw 2021

https://doi.org/10.15632/jtam-pl/134281

\title{
STOCHASTIC P-BIFURCATION OF A 3-DOF AIRFOIL WITH STRUCTURAL NONLINEARITY
}

\author{
Ying HaO, Xuewen Du, Yuda Hu \\ College of Civil Engineering and Mechanics, Yanshan University, Qinhuangdao, China \\ e-mail: haoying@tju.edu.cn \\ ZHIQIANG WU \\ School of Mechanical Engineering, Tianjin University, Tianjin, China
}

\begin{abstract}
In this paper, the stochastic-aeroelastic nonlinear response of a three-degree-of-freedom (3-DOF) structural nonlinear airfoil with a control flap is presented. The critical parameter conditions of stochastic P-bifurcation are solved by using the improved average method, the stochastic average method combined with the singularity theory. The results show that the periodic solution produced by Hopf bifurcation has involved a second bifurcation, the nonlinear critical speed of saddle node bifurcation points is advanced, and the airfoil appears bi-stable. The stochastic singularity analysis shows that the increasing stochastic disturbance intensity will cause a greater probability for a large amplitude stochastic flutter.
\end{abstract}

Keywords: stochastic P-bifurcation, structural nonlinearity, stochastic parametric excitation

\section{Introduction}

Structural nonlinearity of the airfoil may lead to complicated nonlinear dynamic behaviour, such as LCO, periodic doubling bifurcation, chaos, and so on. As present, the LCO and chaos response of a two-dimensional wing with control surface nonlinearity were studied by Virgin and Dowell (1992). Conner et al. (1997) investigated theoretically piecewise linear state-space models for a 3-DOF aero-elastic typical section with control surface freeplay, which was validated by an experimental model of a 3-DOF typical section in the two-dimensional space. The flutter of a 3-DOF wing with cubic nonlinear stiffness in incompressible flow was studied by using Hopf bifurcation theory by Zheng and Yang (2006). The results showed that there was a subcritical Hopf bifurcation and the flutter critical speed decreased versus the increasing linear stiffness coefficient. Irani et al. (2011) analyzed a 3-DOF aeroelastic typical section with a trailing-edge control surface, and the numerical analysis revealed the presence of stable and unstable limit cycles along with stability reversal in the neighborhood of a Hopf bifurcation. The LCO of two models of the wing/store with free play in the junction, one of a two-dimensional wing, and the other a delta wing, were investigated by Yang (1995). The KBM method was used to analyze the limit cycle flutter of the wing, and also, the results were compared with the wind-tunnel test results. Other experimental studies of LCO of the airfoil can be found in the works by Dowell et al. (2004) and Chassaing et al. (2012).

Besides, some scholars introduced higher-order nonlinearity to investigate the multi-stable characteristics (hard-destabilization) of airfoil flutter. Christiansen et al. (2002) considered two nonlinear models: an extended normal-form model and a nonlinear version of 2-DOF structural models. Both models yield the same discovery. The subcritical Hopf bifurcation and the saddle-node bifurcation were observed by Poirel and Price (2007). At the interval between these two bifurcations, the unstable limit cycle represents a separatrix between the equilibrium state and the fluttering state. Missoum et al. (2010) introduced a methodology for the reliability-based 
design optimization of systems with nonlinear aeroelastic constraints. The proposed approach was applied to the generation of flutter and subcritical LCO boundary finding a 2-DOF airfoil with a fifth-order nonlinear stiffness. Moreover, Dribusch et al. (2010) also considered the quantic nonlinearity stiffness when studying the stability boundary of aeroelastic problems. Hao and $\mathrm{Wu}$ $(2019,2020)$ considered a quintic nonlinearity of the torsional spring, and showed that the pitchfork bifurcation occurred.

Current research of airfoil flutter systems under random perturbation is not all-inclusive yet; few authors have turned their eyes to random perturbation and airfoil multi-stability. In the 1990s, Poirel and Price (1997, 2003b, 2012), Poirel et al. (2005) and Lee et al. (1999) and his colleagues pioneered researches on turbulence-induced stochastic flutter by addressing the existence of stochastic turbulent perturbation in their studies. Poirel and Price (2007) pioneered the stochastic bifurcation study of the airfoil flutter system by looking at the effects of stochastic P-bifurcation of a binary airfoil model. Poirel and Price (1997) examined the stability of an airfoil model with structural nonlinearity in the pitch direction by incorporating the effects of turbulence into the longitudinal inflow velocity. A fairly complete description of airfoil flutter with structural or aerodynamic nonlinearity was involved by Lee et al. (1999), and numerically investigated the effects of longitudinal turbulent perturbation on Hopf bifurcation and airfoil flutter boundary. Poirel and Price (2003a) addressed the effects of both longitudinal and vertical turbulence in their airfoil model.

The larger amplitude of LCO flutter induced by subcritical Hopf bifurcation, which occours before the linear critical flutter speed, may infect flying security, where the jumping of nonlinear solutions increases the difficulty of traditional optimization design and critical speed prediction. Since the airfoil flutter system may exhibit secondary bifurcation and multi-steady state due to high-order structural nonlinearity, in this paper, the high-order quantic structural nonlinearity is introduced to investigate nonlinear flutter of the 3-DOF airfoil . By combining the numerical method and the stochastic P-bifurcation analysis for high dimensional systems, the influence of parameters variation on the linear and nonlinear critical flutter speed is discussed.

\section{Equations of motion}

Consider a 3-DOF airfoil with a control surface (flap) and a nonlinear torsional spring (Zheng and Yang, 2006; Irani et al., 2011). The plunging deflection is denoted by $h, \alpha$ is the pitch angle, and $\beta$ is the flap angle, positive when the trailing edge surface is moved down. The elastic axis is located at a distance $a_{h} b$ from the mid-chord, where $b$ is half of the chord. The wing mass center is located at a distance $x_{\alpha} b$ from the elastic axis. The axis of rotation of the flap is located at a distance $c_{h} b$ from the mid-chord, while the flap mass center is located at a distance $x_{\beta} b$ from the flap hinge. All the previous distances are positive when measured towards the flap of the airfoil.

Based on Theodorsen's linear aerodynamic model in (Theodorsen, 1935), the aeroelastic equation of motion for nonlinear torsional springs are originally found by Irani et al. (2011) as follows

$$
\begin{aligned}
& m \ddot{h}+S_{\alpha} \ddot{\alpha}+S_{\beta} \ddot{\beta}+K_{h} h=L \\
& S_{\alpha} \ddot{h}+J_{\alpha} \ddot{\alpha}+\left[J_{\beta}+b\left(c_{h}-a_{h}\right)\right] \ddot{\beta}+K_{\alpha}\left[k_{1 \alpha} \alpha+M(\alpha)\right]=M_{\alpha} \\
& S_{\beta} \ddot{h}+\left[J_{\beta}+b\left(c_{h}-a_{h}\right)\right] \ddot{\alpha}+J_{\beta} \ddot{\beta}+K_{\beta}\left[k_{1 \beta} \beta+M(\beta)\right]=M_{\beta}
\end{aligned}
$$

where $K_{\alpha}, K_{h}, K_{\beta}$ - linear stiffness coefficients at $\alpha, h$ and $\beta ; S_{\alpha}, S_{\beta}$ - airfoil static moments about the elastic axis and the flap hinge; $J_{\alpha}, J_{\beta}$ - airfoil mass moments of inertia about the elastic axis and the flap hinge. 
Our study ignored the aerodynamic force, aerodynamic moment acting on the flap, hence $M_{\beta}$ was not considered $\left(M_{\beta}=0\right) . M(\alpha)$ and $M(\beta)$ are the nonlinear pitch and flap stiffness terms. For incompressible flow, the governing equations of the airfoil system are established on quasi-steady aerodynamics. The aerodynamic lift force and the aerodynamic moment are given by Zheng and Yang (2006)

$$
\begin{aligned}
& L=-2 \pi \rho b Q^{*}\left[\alpha Q^{*}+\dot{h}+b\left(\frac{1}{2}-a_{h}\right) \dot{\alpha}\right] \\
& M_{\alpha}=2 \pi \rho Q^{*} b^{2}\left(\frac{1}{2}+a_{h}\right)\left[\alpha Q^{*}+\dot{h}+b\left(\frac{1}{2}-a_{h}\right) \dot{\alpha}\right]-\frac{1}{2} \pi \rho Q^{*} b^{3} \dot{\alpha}
\end{aligned}
$$

where $\rho$ is mass of the air per unit of volume, $Q^{*}$ is generalized flow speed, the stochastic disturbance $u_{T}^{*}$ is considered in $Q^{*}$, we assume that $Q^{*}=Q_{m}^{*}+u_{T}^{*}, u_{T}^{*}$ is the stochastic disturbance.

In the particular case, we only define high order structural nonlinearity in the pitch degree of freedom $\left(M(\alpha)=k_{3 \alpha} \alpha^{3}-k_{5 \alpha} \alpha^{5}\right)$, where $M(\beta)=0$ and $k_{3 \alpha}, k_{5 \alpha}$ are nonlinear stiffness coefficients. Specify the following non-dimensional coefficients by these definitions

$$
\begin{array}{lccrr}
\xi=\frac{h}{b} & x_{\alpha}=\frac{S_{\alpha}}{m b} & x_{\beta}=\frac{S_{\beta}}{m b} & \omega_{h}^{2}=\frac{K_{h}}{m} & \omega_{\alpha}^{2}=\frac{K_{\alpha}}{J_{\alpha}} \\
\omega_{\beta}^{2}=\frac{K_{\beta}}{J_{\beta}} & r_{\alpha}^{2}=\frac{J_{\alpha}}{m b^{2}} & r_{\beta}^{2}=\frac{J_{\beta}}{m b^{2}} & u=\frac{m}{\rho \pi b^{2}} & Q_{m}=\frac{Q_{m}^{*}}{b \omega_{\alpha}} \\
u_{T}=\frac{u_{T}^{*}}{b \omega_{\alpha}} & \omega_{1}=\frac{\omega_{h}}{\omega_{\alpha}} & \omega_{2}=\frac{\omega_{\beta}}{\omega_{\alpha}} & \tau=\frac{Q_{m}^{*} t}{b} &
\end{array}
$$

where $r$ is the aeroelastic modal amplitude, $r_{\alpha}, r_{\beta}, r_{\xi}$ amplitude of $\alpha, \beta$ and $\xi$.

We obtain the system of $\xi, \dot{\xi}, \alpha, \dot{\alpha}, \beta, \dot{\beta}$, let $x_{1}=\dot{\xi}, x_{2}=\dot{\xi}, x_{3}=\alpha, x_{4}=\dot{\alpha}, x_{5}=\beta, x_{6}=\dot{\beta}$, then Eqs. (2.1) could be expressed as

$$
\begin{aligned}
& \dot{x}_{1}=x_{2} \\
& \dot{x}_{2}=a_{21} x_{1}+a_{22} x_{2}+a_{23} x_{3}+a_{24} x_{4}+a_{25} x_{5}+a_{26} x_{6}+a_{27} M\left(x_{3}\right) \\
& \dot{x}_{3}=x_{4} \\
& \dot{x}_{4}=a_{41} x_{1}+a_{42} x_{2}+a_{43} x_{3}+a_{44} x_{4}+a_{45} x_{5}+a_{46} x_{6}+a_{47} M\left(x_{3}\right) \\
& \dot{x}_{5}=x_{6} \\
& \dot{x}_{6}=a_{61} x_{1}+a_{62} x_{2}+a_{63} x_{3}+a_{64} x_{4}+a_{65} x_{5}+a_{66} x_{6}+a_{67} M\left(x_{3}\right)
\end{aligned}
$$

where

$$
\begin{aligned}
a_{21} & =\frac{e \omega_{1}^{2}}{Q_{m}^{2}}\left(b_{2} c_{3}-b_{3} c_{2}\right) \quad a_{22}=\frac{2 e\left(\frac{1}{2}+a_{h}\right) U_{1}}{u r_{\alpha}^{2}}\left(a_{2} c_{3}-a_{3} c_{2}\right)+\frac{2 e U_{1}}{u}\left(b_{2} c_{3}-b_{3} c_{2}\right) \\
a_{23} & =\frac{2 e\left(\frac{1}{2}+a_{h}\right) U_{2}}{u r_{\alpha}^{2}}\left(a_{2} c_{3}-a_{3} c_{2}\right)+\frac{e k_{1 \alpha}}{Q_{m}^{2}}\left(a_{3} c_{2}-a_{2} c_{3}\right)+\frac{2 e U_{2}}{u}\left(b_{2} c_{3}-b_{3} c_{2}\right) \\
a_{24} & =\frac{1}{2} \frac{e U_{1}}{u r_{\alpha}^{2}}\left(a_{3} c_{2}-a_{2} c_{3}\right)+\frac{2 e\left(\frac{1}{2}-a_{h}\right)\left(\frac{1}{2}+a_{h}\right) U_{1}}{u r_{\alpha}^{2}}\left(a_{2} c_{3}-a_{3} c_{2}\right) \\
& +\frac{2 e\left(\frac{1}{2}-a_{h}\right) U_{1}}{u}\left(b_{2} c_{3}-b_{3} c_{2}\right) \\
a_{25} & =\frac{e \omega_{2}^{2}}{Q_{m}^{2}}\left(a_{2} b_{3}-a_{3} b_{2}\right) \quad a_{26}=0 \quad \frac{e}{Q_{m}^{2}}\left(a_{3} c_{2}-a_{2} c_{3}\right) \\
a_{41} & =\frac{e \omega_{1}^{2}}{Q_{m}^{2}}\left(b_{1} c_{3}-b_{3} c_{1}\right) \quad a_{42}=\frac{2 e\left(\frac{1}{2}+a_{h}\right) U_{1}}{u r_{\alpha}^{2}}\left(a_{1} c_{3}-a_{3} c_{1}\right)+\frac{2 e U_{1}}{u}\left(b_{1} c_{3}-b_{3} c_{1}\right)
\end{aligned}
$$




$$
\begin{aligned}
& a_{43}=\frac{2 e\left(\frac{1}{2}+a_{h}\right) U_{2}}{u r_{\alpha}^{2}}\left(a_{1} c_{3}-a_{3} c_{1}\right)+\frac{e k_{1 \alpha}}{Q_{m}^{2}}\left(a_{3} c_{1}-a_{1} c_{3}\right)+\frac{2 e U_{2}}{u}\left(b_{1} c_{3}-b_{3} c_{1}\right) \\
& a_{44}=\frac{1}{2} \frac{e U_{1}}{u r_{\alpha}^{2}}\left(a_{3} c_{1}-a_{1} c_{3}\right)+\frac{2 e\left(\frac{1}{2}-a_{h}\right)\left(\frac{1}{2}+a_{h}\right) U_{1}}{u r_{\alpha}^{2}}\left(a_{1} c_{3}-a_{3} c_{1}\right) \\
& +\frac{2 e\left(\frac{1}{2}-a_{h}\right) U_{1}}{u}\left(b_{1} c_{3}-b_{3} c_{1}\right) \\
& a_{45}=\frac{e \omega_{2}^{2}}{Q_{m}^{2}}\left(a_{1} b_{3}-a_{3} b_{1}\right) \quad a_{46}=0 \quad a_{47}=\frac{e}{Q_{m}^{2}}\left(a_{3} c_{1}-a_{1} c_{3}\right) \\
& a_{61}=\frac{e \omega_{1}^{2}}{Q_{m}^{2}}\left(b_{1} c_{2}-b_{2} c_{1}\right) \quad a_{62}=\frac{2 e\left(\frac{1}{2}+a_{h}\right) U_{1}}{u r_{\alpha}^{2}}\left(a_{1} c_{2}-a_{2} c_{1}\right)+\frac{2 e U_{1}}{u}\left(b_{1} c_{2}-b_{2} c_{1}\right) \\
& a_{63}=\frac{2 e\left(\frac{1}{2}+a_{h}\right) U_{2}}{u r_{\alpha}^{2}}\left(a_{1} c_{2}-a_{2} c_{1}\right)+\frac{e k_{1 \alpha}}{Q_{m}^{2}}\left(a_{2} c_{1}-a_{1} c_{2}\right)+\frac{2 e U_{2}}{u}\left(b_{1} c_{2}-b_{2} c_{1}\right) \\
& a_{64}=\frac{1}{2} \frac{e U_{1}}{u r_{\alpha}^{2}}\left(a_{1} c_{1}-a_{1} c_{2}\right)+\frac{2 e\left(\frac{1}{2}-a_{h}\right)\left(\frac{1}{2}+a_{h}\right) U_{1}}{u r_{\alpha}^{2}}\left(a_{1} c_{2}-a_{2} c_{1}\right) \\
& +\frac{2 e\left(\frac{1}{2}-a_{h}\right) U_{1}}{u}\left(b_{1} c_{2}-b_{2} c_{1}\right) \\
& a_{65}=\frac{e \omega_{2}^{2}}{Q_{m}^{2}}\left(a_{1} b_{2}-a_{2} b_{1}\right) \quad a_{66}=0 \quad a_{67}=\frac{e}{Q_{m}^{2}}\left(a_{2} c_{1}-a_{1} c_{2}\right) \\
& e=\frac{1}{a_{1} b_{2} c_{3}-a_{1} b_{3} c_{2}-a_{2} b_{1} c_{3}+a_{2} b_{3} c_{1}+a_{3} b_{1} c_{2}-a_{3} b_{2} c_{1}} \\
& a_{1}=1 \quad a_{2}=x_{\alpha} \quad a_{3}=x_{\beta} \\
& b_{1}=\frac{x_{\alpha}}{r_{\alpha}^{2}} \quad b_{2}=1 \quad b_{3}=\left[r_{\beta}^{2}+x_{\beta}\left(c_{h}-a_{h}\right)\right] \frac{1}{r_{\alpha}^{2}} \\
& c_{1}=\frac{x_{\beta}}{r_{\beta}^{2}} \quad c_{2}=1+\frac{x_{\beta}}{r_{\beta}^{2}}\left(c_{h}-a_{h}\right) \quad c_{3}=1 \\
& U_{1}=\frac{Q(\tau)}{Q_{m}} \quad U_{2}=\left(\frac{Q(\tau)}{Q_{m}}\right)^{2}
\end{aligned}
$$

\section{Power density distribution of 3-DOF aeroelastic modal amplitude}

Equations (2.3) can be expressd as

$$
\dot{\mathbf{x}}=F\left(x, Q_{m}, \tau\right)
$$

here the inflow speed $Q_{m}$ is a bifurcation parameter under which the bifurcation point of a deterministic system is $\left(x, Q_{m}\right)=\left(0, Q_{m 0}\right)$, and $Q_{m 0}$ is the bifurcation critical point. Let $Q_{m}=Q_{m 0}+u$. It is defined that the perturbation near the Hopf bifurcation point is $u=\mu+u_{T}$, where $\mu$ is the introduced small parameter, $u_{T}(\tau)$ is the stochastic disturbance. Hence, the Jacobian matrix concerning perturbation can be expressed as

$$
\mathbf{A}(u)=\mathbf{A}(0, u, 0)=\left.\frac{\partial F}{\partial x}\right|_{\substack{x=0 \\ Q=Q_{m 0}+u \\ t=0}}
$$


Expanding Eq. (3.2) at $u=0$, yields $\mathbf{A}(u)=\mathbf{A}_{0}+\left.D \mathbf{A}(u)\right|_{u=0} u=\mathbf{A}_{0}+\mathbf{A}_{1} u$, Hence, Eq. (3.1) can be rewritten as

$$
\dot{\mathbf{x}}=\left(\mathbf{A}_{0}+\mathbf{A}_{1} u\right) \mathbf{x}+\left[F\left(x_{i}, Q_{m}, t\right)-\left(\mathbf{A}_{0}+\mathbf{A}_{1} u\right) \mathbf{x}\right]=\mathbf{A}_{0} \mathbf{x}+\mathbf{F}_{a}\left(x_{i}, u\right)
$$

where $\mathbf{F}_{a}\left(x_{i}, u\right)$ are the quadratic polynomials. Assume that the solution of the derived system $\left(\dot{\mathbf{x}}=\mathbf{A}_{0} \mathbf{x}\right)$ is

$$
\mathbf{x}=\mathbf{G}(\tau) \mathbf{b}
$$

where $\mathbf{G}(\tau)=\mathbf{T E}(\tau)$ is the fundamental matrix of Eq. (3.1), where $\mathbf{E}(\tau)=$ $\operatorname{diag}\left(\mathrm{e}^{\lambda_{1} \tau}, \mathrm{e}^{\lambda_{2} \tau}, \mathrm{e}^{\lambda_{3} \tau}, \mathrm{e}^{\lambda_{4} \tau}, \mathrm{e}^{\lambda_{5} \tau}, \mathrm{e}^{\lambda_{6} \tau}\right), \mathbf{T}=\left[\varphi_{1}, \varphi_{2}, \varphi_{3}, \varphi_{4}, \varphi_{5}, \varphi_{6}\right]$, and $b$ is a function of $\tau$. $\lambda_{j}$ is the eigenvalue of Jacobian matrix, $\lambda_{1}, \lambda_{2}$ are the pair of pure imaginary numbers, and the other four $\left(\lambda_{3}, \ldots, \lambda_{6}\right)$ have negative real parts, $\varphi_{j}$ is the eigenvector corresponding to each eigenvalue.

By substituting Eq. (3.4) into Eq. (3.3) from the method of variation of constants, we have

$$
\dot{\mathbf{b}}=\left.\mathbf{G}^{*}(\tau) \mathbf{F}_{a}\left(x_{i}, u\right)\right|_{\mathbf{x}=\mathbf{G}(\tau) \mathbf{b}}=\mathbf{H}\left(b_{i}, u, \tau\right)=\left[H_{1}, H_{2}, H_{3}, H_{4}, H_{5}, H_{6}\right]^{\mathrm{T}}
$$

where $\mathbf{G}^{*}(\tau)$ is the inverse of the fundamental matrix $\mathbf{G}(\tau)$. Suppose $\mathbf{b}$ are functions of $\tau$, which is convenient to derive the solution in a more familiarized familiar form

$$
\begin{aligned}
b_{1} & =r \mathrm{e}^{\mathrm{i} \theta} & b_{2} & =r \mathrm{e}^{-\mathrm{i} \theta} \\
b_{i} & =b_{i}(\tau) & i & =3, \ldots, 6
\end{aligned}
$$

where $r$ is the aeroelastic modal amplitude, $\theta$ is generalized phase-angle.

By making use of the stochastic averaging and modified averaging method (Hao and Wu, 2019, 2020), when $\tau$ approaches to infinity, $b_{i}=0(i=3, \ldots, 6)$, we obtain the normal equation by substituting Eq. (3.6) into Eq.(3.5)

$$
\dot{r}=H_{1} \cos \theta+H_{2} \sin \theta=\Phi(r, \theta, u) \quad \dot{\theta}=\frac{-H_{1} \sin \theta+H_{2} \cos \theta}{r_{j}}=\Phi^{*}(r, \theta, u)
$$

where

$$
\begin{aligned}
\Phi(r & , \theta, u)=-[-1.2788 \cos (4.5624 \tau-4 \theta)+1.1184 \sin (2.2812 \tau-2 \theta)+0.0315 \\
& -0.2518 \sin (6.8436 \tau-6 \theta)-0.1983 \cos (6.8436 \tau-6 \theta)-0.0905 \sin (4.5624 \tau-4 \theta) \\
& -1.1484 \cos (2.2812 \tau-2 \theta)] k_{5 \alpha} r^{5}+[0.3319 \sin (2.2812 \tau-2 \theta)-0.0181 \sin (4.5624 \tau-4 \theta) \\
& -0.3475 \cos (2.2812 \tau-2 \theta)+0.071-0.2395 \cos (4.5624 \tau-4 \theta)] k_{3 \alpha} r^{3} \\
& +[0.0005+0.6777 \cos (2.2812 \tau-2 \theta)-0.5706 \sin (2.2812 \tau-2 \theta)] \mu r \\
& +[0.0099 \sin (2.2812 \tau-2 \theta)-0.0114 \cos (2.2812 \tau-2 \theta)-0.0004] \mu r u_{T}^{2}(\tau) \\
& +[0.0121 \sin (2.2812 \tau-2 \theta)-0.0156 \cos (2.2812 \tau-2 \theta)+0.0005] \mu r u_{T}(\tau) \\
\Phi^{*} & (r, \theta, u)=-[-0.2518 \cos (6.8436 \tau-6 \theta)+3.2046-0.1514 \cos (4.5624 \tau-4 \theta) \\
& +1.9169 \sin (4.5624 \tau-4 \theta)+0.1983 \sin (6.8436 \tau-6 \theta)+3.5304 \sin (2.2812 \tau-2 \theta) \\
& +3.2625 \cos (2.2812 \tau-2 \theta)] k_{5 \alpha} r^{4}+[0.7046 \sin (2.2812 \tau-2 \theta)+0.7206 \\
& +0.2395 \sin (4.5624 \tau-4 \theta)-0.1814 \cos (4.5624 \tau-4 \theta)+0.6533 \cos (2.2812 \tau-2 \theta)] k_{3 \alpha} r^{2} \\
& +[-0.8861-0.5706 \cos (2.2812 \tau-2 \theta)-0.6777 \sin (2.2812 \tau-2 \theta)] \mu \\
& +[0.9902 \cos (2.2812 \tau-2 \theta)+0.11434 \sin (2.2812 \tau-2 \theta)+0.1512] \mu u_{T}^{2}(\tau) \\
& +[0.1214 \cos (2.2812 \tau-2 \theta)+0.1558 \sin (2.2812 \tau-2 \theta)+0.1925] \mu u_{T}(\tau)
\end{aligned}
$$


The new normal equation is about $r$ and $\theta$. By substituting $\mathbf{G}(t)$ and $\mathbf{b}$ into $\mathbf{x}=\mathbf{G}(t) \mathbf{b}$, we have the expression of $\mathbf{x}$, where $\varphi=1.1406 \tau-\theta$

$$
\mathbf{x}=\left[\begin{array}{c}
\xi \\
\dot{\xi} \\
\alpha \\
\dot{\alpha} \\
\beta \\
\dot{\beta}
\end{array}\right]=\left[\begin{array}{c}
1.2774 r \cos \varphi+0.6538 r \sin \varphi \\
0.7457 r \cos \varphi-1.4571 r \sin \varphi \\
-2.0884 r \cos \varphi-0.9990 r \sin \varphi \\
-1.1394 r \cos \varphi+2.3821 r \sin \varphi \\
-1.2614 r \cos \varphi-0.5714 r \sin \varphi \\
-0.6518 r \cos \varphi+1.4387 r \sin \varphi
\end{array}\right]
$$

The stochastic average and deterministic average can be utilized in Eqs. (3.7), and we obtain the following stochastic equations for the aeroelastic modal amplitude $r$ and generalized phase-angle $\theta$

$$
\begin{aligned}
d r & =\left[-0.0320 k_{5 \alpha} r^{5}+0.0072 k_{3 \alpha} r^{3}+\left(5.4284 \cdot 10^{-4} D \mu^{2}+1.7946 \cdot 10^{-4} \mu\right) r\right] d \tau \\
& +\sqrt{3.6238 \cdot 10^{-4} r^{2} \mu^{2} D} d W_{1}(\tau) \\
d \theta= & \left(-3.0853 k_{5 \alpha} r^{4}+0.6908 k_{3 \alpha} r^{2}-0.8259 \mu\right) d \tau+\sqrt{0.0010 D \mu^{2}} d W_{2}(\tau)
\end{aligned}
$$

where $D$ is the intensity of stochastic disturbance $u_{T}, W_{1}(t)$ and $W_{2}(t)$ are two independent Wiener processes. The probability density function $p(r, \tau)$ of the instantaneous aeroelastic modal amplitude $r$ satisfies the FPK equation

$$
\begin{gathered}
\frac{\partial p(r, \tau)}{\partial t}=-\frac{\partial}{\partial r}\left\{\left[-0.0320 k_{5 \alpha} r^{5}+0.0072 k_{3 \alpha} r^{3}+\left(5.4284 \cdot 10^{-4} D \mu^{2}\right.\right.\right. \\
\left.\left.\left.\quad+1.7946 \cdot 10^{-4} \mu\right) r\right] p(r, \tau)\right\}+\frac{1}{2} \frac{\partial^{2}}{\partial r^{2}}\left[3.6238 \cdot 10^{-4} r^{2} \mu^{2} D p(r, \tau)\right]
\end{gathered}
$$

By solving the FPK equation, we obtain the stationary probability density function

$$
p_{s}(r)=\kappa \frac{2759.56}{\mu D} r^{0.9960+\frac{0.9905}{\mu D}} \exp \left(\frac{-3.4494 r^{4}+3.4494 r^{2}}{\mu^{2} D}\right)
$$

where $\kappa$ is the unified coefficient, and the system stationary response $\xi, \dot{\xi}, \alpha, \dot{\alpha}, \beta, \dot{\beta}$ and modal amplitude are shown in Eq. (3.8).

\section{Anlysis of the critical flutter speed}

Figure 1 represents bifurcation of the deterministic system, Eq. (2.3), since the high order nonlinearity term $M(\alpha)=k_{3 \alpha} \alpha^{3}-k_{5 \alpha} \alpha^{5}$ exists, which causes the second bifurcation and a bi-stable steady state of the system. Here, the solid line, dotted line, blue stars are the equilibrium, unstable response and the corresponding numerical results, respectively. It can be see that the analytical solution approaches to the numerical solution by adopting the Runge-Kutta fourth-order algorithm.

The main parameter involved in the airfoil flutter is the critical folw speed, which is the critical value of $Q_{m}$. In Fig. 1, the critical speed of Hopf bifurcation $Q_{H}=1.348$ is then defined as the linear critical flutter speed. Moreover, the critical speed of saddle node bifurcation $Q_{A}=0.478$ is defined as the nonlinear critical flutter speed. In Fig. 1, it can be seen that the nonlinear critical flutter speed is less than its linear counterpart. However, in the 3-DOF airfoil with structural nonlinearity in the pitch degree of freedom, even below the linear flutter speed, we encounter large amplitude oscillations. 


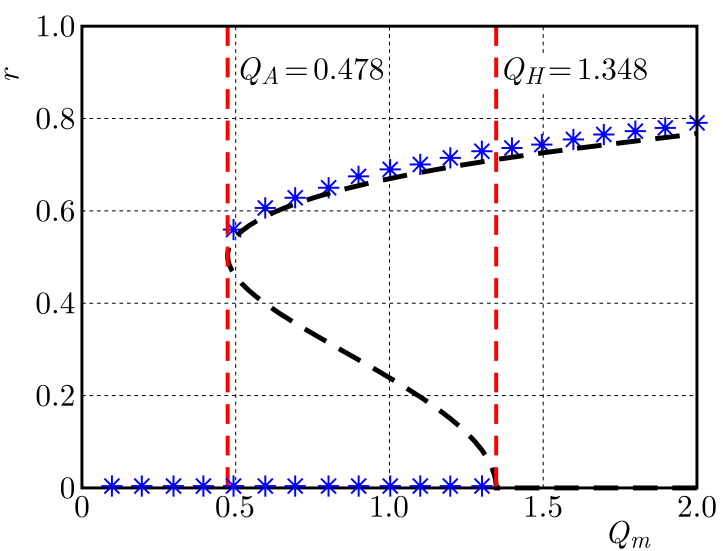

Fig. 1. Bifurcation plot for the aeroelastic modal amplitude $r$ with $u=100, x_{\alpha}=0.25, x_{\beta}=0.0125$, $r_{\alpha}=0.5, r_{\beta}=0.079, \omega_{1}=1.2, \omega_{2}=3.5, M_{\beta}=0, M(\alpha)=0.1746 \alpha^{3}-0.0782 \alpha^{5}$

The linear critical flutter speed $Q_{H}$ can be determined by linear stability analysis, whereas the nonlinear critical flutter speed $Q_{A}$ can only be determined by nonlinear analysis. The effects of high order nonlinearity $k_{3 \alpha}$ and $k_{5 \alpha}$ in the pitching degree on the the nonlinear critical flutter speed $Q_{A}$ are obtained through the averaging method. From Fig. 2a, it can be seen that with the increasing $k_{3 \alpha}$ in the pitching degree of freedom, the value of the nonlinear critical flutter speed $Q_{A}$ decreases. In addition, the nonlinear critical flutter speed $Q_{A}$ increases with a growth in the value of $k_{5 \alpha}$ in the pitching degree of freedom (Fig. $2 \mathrm{~b}$ ).

(a)

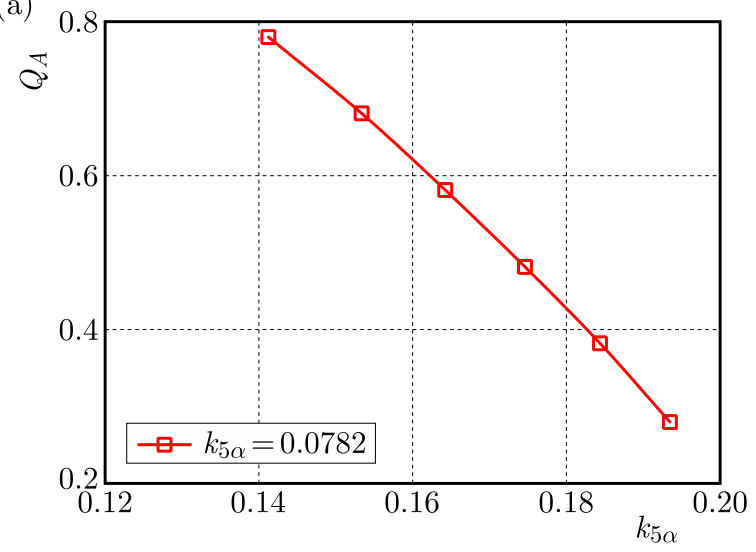

(b)

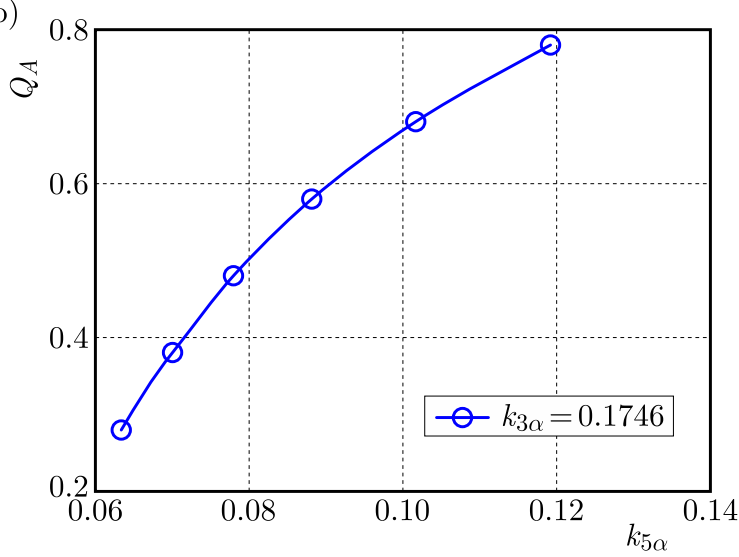

Fig. 2. The cruve of the nonlinear flutter speed $Q_{A}$ varying with: (a) $k_{3 \alpha}$, (b) $k_{5 \alpha}$

\section{Stochastic P-bifurcation analysis of the stochastic system}

For this non-linear 3-DOF airfoil, we analyze stationary response behavior of the system by investigating the standard equations of the aeroelastic modality amplitude $r$ and $\theta$. First, the bifurcation equation is selected as

$$
g(r)=R(r, D, \mu) \exp [Q(r, D, \mu)]-p_{s}(r)=0
$$

where

$$
R(r, D, \mu)=\frac{2759.56}{\mu D} r^{0.9960+\frac{0.9905}{\mu D}} \quad Q(r, D, \mu)=\frac{-3.4494 r^{4}+3.4494 r^{2}}{\mu^{2} D}
$$

The transition set $H$ (hysteresis set) of the non-linear flutter system corresponding to Eq. (4.1) is solved by using the singularity theory. Types of the transition sets and their calculation 
methods are the same as those in (Hao and $\mathrm{Wu}, 2019)$. Here, the aeroelastic modality amplitude $r$ is a state variable; the stationary probability density $p_{s}(r)$ is a bifurcation parameter; the intensity $D$ of the stochastic disturbance, the perturbation $\mu$ of the inflow speed $Q_{m}$ near the Hopf bifurcation point are unfolding parameters.

Figure 4 shows the transition set profile in $\left(D, Q_{m}\right)$. Here, the dotted line is the hysteresis set $H_{2}$, and the dot-and-dash line is the hysteresis set $H_{1}$. The transition sets $H_{1}$ and $H_{2}$ divide the parameter plane $\left(D, Q_{m}\right)$ into different regions marked as $1,2,3$.

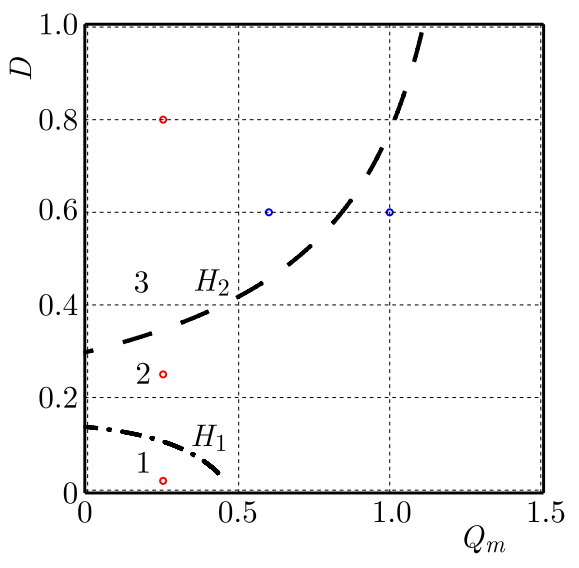

Fig. 3. The critical condition of P-bifurcation (transition sets)

(a)

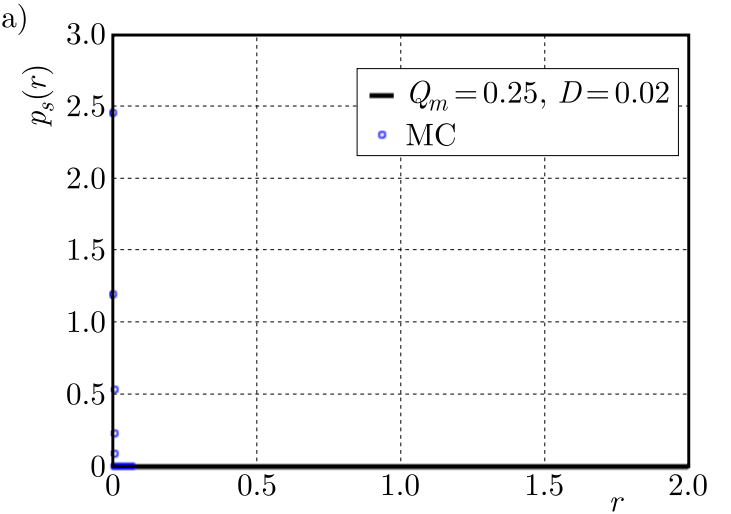

(b)

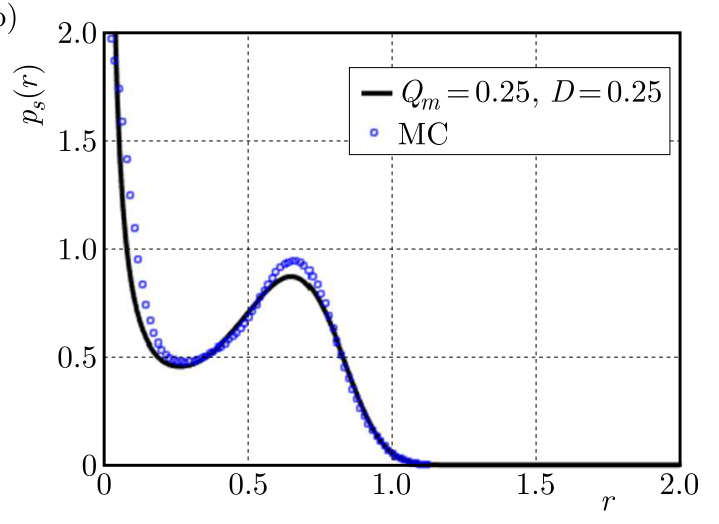

(c)

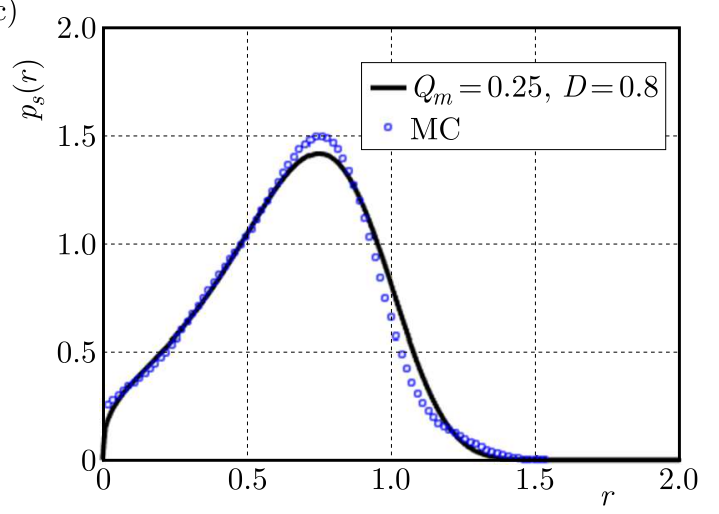

Fig. 4. PDF curves of the aeroelastic modal amplitude $r$ for different regions in Fig. 3

Figure 4 shows the stationary PDF of the aeroelastic modal amplitude $r$ yielded at points selected in different reginons divided by the transition sets (in Fig. 3). Here, the black curve and the blue asterisked line are theoretical results and Monte Carlo (MC) simulation results, 
respectively. From Fig. 4, it can be see that the theoretical calculations agree well with the numerical results which we ran with the Monte Carlo simulation with the sample number set as $10^{6}$.

The PDF of the aeroelastic modal amplitude $r$ obtained from region 1 (in Fig. 3) of the $\left(D, Q_{m}\right)$ parameter plane is shown in Fig. 4a. No large amplitude vibration can be observed in this region, the response refers to a zero solution and a steady-state.

An obvious peak appears in the amplitude $r$ in the PDF curve when the parameter set passes through region 1 to region 2 (in Fig. 3 ) of the $\left(D, Q_{m}\right)$ plane, as shown in Fig. 4b. Large amplitude vibration can be found, but small amplitude vibration still exists.

With the increase of stochastic intensity $D$, passing from region 2 to 3 in Fig. 3, the second stochastic P-bifurcation appears, and only one peak in the PDF curve could be observed. Its probabilistic curve is shown in Fig. 4c. Large amplitude vibration could be observed on the airfoil, and it corresponds to the steady responses of the system.

Through the analysis of the transition sets and the bifurcation curves, the steady response of the system depends on the value of $\left(D, Q_{m}\right)$ in the diagram. When $\left(D, Q_{m}\right)$ obtained from region 1 (in Fig. 3), the response behavior appears to be like the balance point in a deterministic system. With the increasing stochastic intensity $D$, the parameter set will cross the hysteresis set $H_{1}$ or even $H_{2}$ and reach to region 3 (in Fig. 3). This crossing is the occurrence of stochastic P-bifurcation. The crossing in this direction is associated with the increasing vibration amplitude until only one peak occurs in the PDF curve, and the system depicts the LCO flutter similar as in the deterministic case. When the flow speed is lower than the critical flutter speed, for instance $Q_{m}=0.25$, we can observe the appearance of large amplitude vibrations in the system with the increase of the intensity $D$ of the stochastic disturbance. Otherwise, the decrease of its intensity can significantly restrain the behavior of substantial vibration of the airfoil.

In Fig. 5a, slight vibration was located in the steady-state response with the increasing flow speed $Q_{m}$ for $D=0.6$. Another scenario could be observed in Fig. 5b when the $\left(D, Q_{m}\right)$ is found in region 2 (in Fig. 3) as $Q_{m}=1.05$. The greater the stochastic intensity $D$ is, the higher peak in the PDF curve could be observed. Hence, the augmentation of stochastic intensity increases the probability of the substantial vibration of the system.

(a)

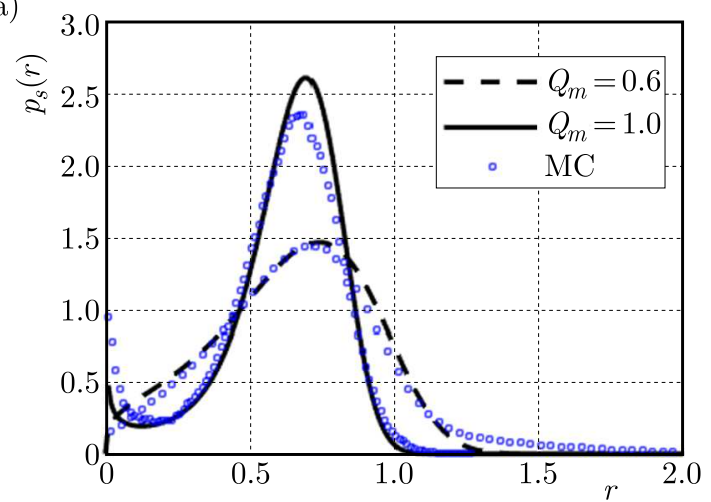

(b)

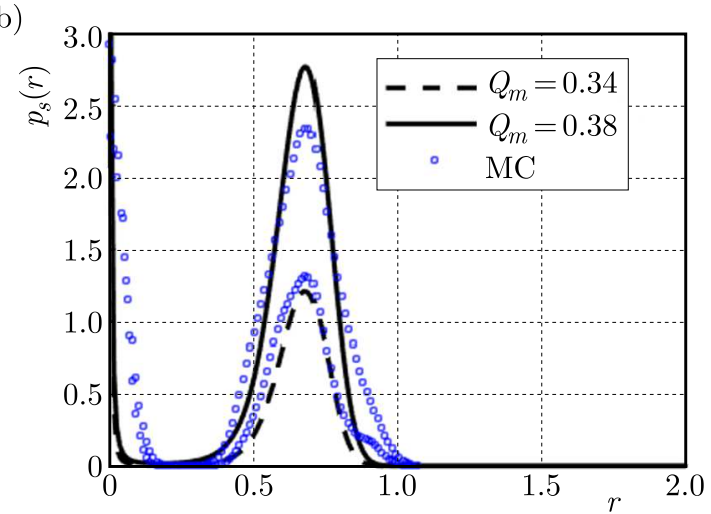

Fig. 5. (a) Influence of $Q_{m}$ on PDF curves of the modal amplitude $r$ at $D=0.6$, (b) influence of $D$ on PDF curves of the modal amplitude $r$ at $Q_{m}=1.05$

\section{Conclusion}

In this work, based on the dimensional multi-steady state stochastic P-bifurcation method, stochastic nonlinear flutter and stochastic P-bifurcation of the multi-stable 3-DOF airfoil in the steady flow with a stochastic disturbance are studied. 
The high order nonlinearity in the pitching degree of freedom causes the secondary saddle-node bifurcation and bi-stable steady states. Meanwhile, large LCO flutter occurs in the system, and the nonlinear critical flutter speed is shifted earlier comparing the linear critical flutter speed of the Hopf bifurcation. Furthermore, the nonlinear critical flutter speed decreases with an invrease of $k_{3 \alpha}$ in the pitching degree of freedom, and increases with an increase of $k_{5 \alpha}$ in the pitching degree of freedom. Moreover, the analysis results show that the large amplitude vibration could still occur along with the increase of the intensity of the stochastic disturbance when the flow speed is inferior to the critical nonlinear flutter speed.

\section{Acknowledgements}

This research is supported by the projects: National Science Foundation of China (No. 11902287), Hebei Provincial Natural Science Foundation of China (No. A2020203007, No. E2020203054).

\section{References}

1. Chassaing J.C., Lucor D., Trégon J., 2012, Stochastic nonlinear aeroelastic analysis of a supersonic lifting surface using an adaptive spectral method, Journal of Sound and Vibration, 331, $2,394-411$

2. Christiansen L.E., Lehn-Schiøler T., Mosekilde E., Gránásy P., Matsushita H., 2002, Nonlinear characteristics of randomly excited transonic flutter, Mathematics and Computers in Simulation, 58, 4-6, 385-405

3. Conner M.D., Tang D., Dowell E., Virgin L., 1997, Nonlinear behavior of a typical airfoil section with control surface freeplay: a numerical and experimental study, Journal of Fluids and Structures, 11, 1, 89-109

4. Dowell E.H., Thomas J.P., Hall K.C., 2004, Transonic limit cycle oscillation analysis using reduced order aerodynamic models, Journal of Fluids and Structures, 19, 1, 17-27

5. Dribusch C., Missoum S., Beran P., 2010, A multifidelity approach for the construction of explicit decision boundaries: application to aeroelasticity, Structural and Multidisciplinary Optimization, 42, 5, 693-705

6. Haо Y., Wu Z.Q., 2019, Random flutter of multi-stable airfoils excited parametrically in steady flows, Journal of Mechanics, 35, 3, 419-426

7. Hao Y., Wu Z., 2020, Stochastic flutter of multi-stable non-linear airfoil in turbulent flow, Journal of Theoretical and Applied Mechanics, 58, 1, 155-168

8. Irani S., Sarrafzadeh H., Amoozgar M.R., 2011, Bifurcation in a 3-DOF airfoil with cubic structural nonlinearity, Chinese Journal of Aeronautics, 24, 3, 265-278

9. LeE B.H.K., Price S.J., Wong Y.S., 1999, Nonlinear aeroelastic analysis of airfoils: bifurcation and chaos, Progress in Aerospace Sciences, 35, 3, 205-334

10. Missoum S., Dribusch C., Beran P., 2010, Reliability-based design optimization of nonlinear aeroelasticity problems, Journal of Aircraft, 47, 3, 992-998

11. Poirel D., Dunn S., Porter J., 2005, Flutter-margin method accounting for modal parameters uncertainties, Journal of Aircraft, 42, 5, 1236-1243

12. Poirel D.C., Price S.J., 1997, Post-instability behavior of a structurally nonlinear airfoil in longitudinal turbulence, Journal of Aircraft, 34, 5, 619-626

13. Poirel D.C., Price S.J., 2003a, Random binary (coalescence) flutter of a two-dimensional linear airfoil, Journal of Fluids and Structures, 18, 1, 23-42

14. Poirel D.C., Price S.J., 2003b, Response probability structure of a structurally nonlinear fluttering airfoil in turbulent flow, Probabilistic Engineering Mechanics, 18, 2, 185-202 
15. Poirel D., Price S.J., 2007, Bifurcation characteristics of a two-dimensional structurally nonlinear airfoil in turbulent flow, Nonlinear Dynamics, 48, 4, 423-435

16. Poirel D.C., Price S.J., 2012, Structurally nonlinear fluttering airfoil in turbulent flow, $A I A A$ Journal, 39, 10, 1960-1968

17. Theodorsen T., 1935, General theory of aerodynamic instability and the mechanism of flutter, NACA Report, 496, 413-433

18. Virgin L., Dowell E.H., 1992, Nonlinear aeroelasticity and chaos, Computational Nonlinear Mechanics in Aerospace Engineering, 1, 531-546

19. YANG Y.R., 1995, KBM method of analyzing limit cycle flutter of a wing with an external store and comparison with a wind-tunnel test, Journal of Sound and Vibration, 187, 2, 271-280

20. Zheng G., YANG Y., 2006, Analysis of bifurcation of an airfoil with an external store in incompressible flow, Science Technology and Engineering, 6, 8, 1018-1021

Manuscript received September 22, 2020; accepted for print February 26, 2021 